\section{Cahiers de Narratologie}

Analyse et théorie narratives

31 | 2016

Sérialité narrative. Enjeux esthétiques et économiques

\title{
Proust en BD : économie de lecture et ré-création proustienne
}

Julie Müller

\section{(2) OpenEdition}

Journals

\section{Electronic version}

URL: https://journals.openedition.org/narratologie/7604

DOI: 10.4000/narratologie.7604

ISSN: 1765-307X

Publisher

LIRCES

\section{Electronic reference}

Julie Müller, "Proust en BD : économie de lecture et ré-création proustienne", Cahiers de Narratologie [Online], 31 | 2016, Online since 22 December 2016, connection on 08 June 2021. URL: http:// journals.openedition.org/narratologie/7604 ; DOI: https://doi.org/10.4000/narratologie.7604

This text was automatically generated on 8 June 2021.

Article L.111-1 du Code de la propriété intellectuelle. 


\title{
Proust en BD : économie de lecture et ré-création proustienne
}

\author{
Julie Müller
}

\section{En guise d'introduction...}

1 Il nous faut d'abord revenir sur une brève définition de la bande dessinée, afin de mieux comprendre par la suite le principe d'économie fondamental qu'elle engage.

2 On peut dire que la bande dessinée est une forme de narration par l'image qui consiste à aligner des scènes dessinées (d'où le terme de "bande ») - qui représentent chronologiquement divers moments de l'action dans le temps. Les personnages peuvent s'exprimer sous forme de bulles qui représentent la parole ou la pensée d'un personnage à un moment précis. De manière réduite, la bande dessinée répond donc à deux principes essentiels: à un principe de réduction, mais aussi à un principe d'aplanissement puisque la bande dessinée fait se dérouler sous les yeux de son lecteur une action continue dans le temps en n'en représentant que quelques instants choisis sous la forme d'icônes, de cases bien définies par leurs limites graphiques mais aussi par les limites imposées par l'espace de la planche.

Or, le temps est, dans l'espace proustien, aux antipodes de toute idée d'aplanissement ou de réduction. La Recherche proustienne est, au contraire, l'incarnation d'un espacetemps qui se déploie, remonte des profondeurs pour finalement venir s'éveiller à la conscience. Il s'agit d'une pensée libre, flottante, qui virevolte dans les abîmes de la mémoire, se laisse aller à des vertiges, faisant des bonds (de parfois plusieurs pages) et qui, après de longues pérégrinations et des détours fortuits, fait enfin affleurer à la surface du texte - et de la conscience - le souvenir, l'image enfin modulée par la mémoire sensible. L'écriture proustienne répond donc à des principes de soudure, de fusion et de verticalité, qui semblent bien loin, voire incompatibles, avec le principe d'horizontalité, la linéarité et les contraintes formelles que dicte la forme séquentielle de la BD. 
On peut alors comprendre pourquoi, quand Stéphane Heuet s'est attaqué il y a environ quinze ans à l'œuvre de Marcel Proust, la critique a d'abord été assassine. "Proust au rabais ", "Proust pour tous ${ }^{1} »$ : Proust en BD ne pouvait être qu'un aplanissement contre-nature de l'écriture proustienne, un détournement du Saint Graal de la littérature française réputé généralement illisible, parfois en bien (d'après certains spécialistes soucieux de préserver un certain élitisme) ou en mal (pour bon nombre de néophytes déroutés par les volutes de cette écriture, et, qui, comme Heuet lui-même au moment de sa première lecture de Proust, doivent avouer que «après la lecture des quinze premières pages de Combray, le livre m'est tombé des mains d'ennui » (Heuet 2012 : 145). Les deux camps semblaient unanimes: mettre Proust en BD relevait du sacrilège. Ce tour de force impossible ne pouvait être qu'un détournement contrenature, motivé par une simple une opération de merchandising.

5 Toutefois, cela était sans compter avec ce néophyte proustien repenti qu'est devenu Stéphane Heuet quelques années plus tard, lui qui dit avoir « redécouvert Proust » par hasard et avoir été frappé, lors de cette nouvelle lecture, par son humour, la justesse concise de ses analyses, et surtout, ce qu'il nomme « un Proust visuel », une écriture qui rompt avec ses limites et qui délivre à son lecteur un véritable graphisme des mouvements de la conscience (Heuet 2012 : 145).

Très vite les anti-Heuet ont reconnu leur faute. Le succès de Proust en bande dessinée est aujourd'hui mondial, commercial certes, mais surtout littéraire, et reconnu par les plus grands spécialistes. Car, comme l'a parfaitement souligné Umberto Eco (1972: 118) :

S'il est vrai que la B.D. se sert à la fois de l'image (et relève par-là de la critique et de l'histoire de l'art) et du texte (qui relève entre autres de la linguistique), il serait faux d'y voir une simple addition de ces deux formes de communication. [...] Le problème consiste plutôt à comprendre comment la fusion de deux modes d'expression distincts produit un langage sui generis aux règles bien distinctes, langage qui a influencé le cinéma, la peinture et jusqu'à la littérature. [...]

7 Le principe d'aplanissement dans l'espace de la bande dessinée cache, en fait, un principe très fortement dialogique. Une polyphonie s'installe entre le texte et l'image au-delà de leurs limites visibles. Car la BD est par définition l'art de la métalepse. La case de bande dessinée est une icône graphique qui ne prend tout son sens que quand on connaît celles qui précèdent et celles qui la suivent. Il s'agit en d'autres termes d'un raccourci référentiel, énonciatif, fondé sur un lien implicite dépassant largement le cadre exigu formé par les contours visibles de la case. La case de bande dessinée fait signe vers des ponts, des passerelles à construire. L'univers séquentiel de la BD exige de son lecteur une participation active afin de recréer l'histoire, de pallier les blancs d'une case à l'autre mais aussi entre les planches. Ce dernier doit effectuer les soudures qui donnent un sens à sa lecture, comme le souligne ici Scott MCcloud (2007 : 75)

Les cases d'une bande dessinée fragmentent à la fois l'espace et le temps, proposant sur un rythme haché des instants qui ne sont pas enchaînés. Mais notre sens de l'ellipse nous permet de relier ces instants et de construire mentalement une réalité globale et continue.

Revenons donc un instant sur cet art de la dislocation inhérent au genre de la BD. Le premier tome de la BD de Stéphane Heuet, va nous permettre, à travers des exemples précis, d'illustrer le principe d'économie fondamental qu'elle engage. Dans un second temps, il s'agira toutefois de relever combien ce très fort principe de réduction dans l'art séquentiel fait appel à une esthétique de la réception qui, loin de trahir l'essence 
de La Recherche proustienne, semble, au contraire, permettre au lecteur de BD de la revivre et de se l'approprier.

\section{Le principe d'économie : réduction et dislocation au sein de l'espace graphique}

dislocation du texte littéraire au sein des différentes planches est, comme nous l'avons vu, inévitable. L'univers séquentiel est restreint dans le monde de la BD et répond aux lois de la coupure, de la brisure, du blanc.

a Recherche proustienne s'étend sur plus de 2000 pages. La première partie - Combray comprend dans l'édition La Pléiade 180 pages - est décrite dans le premier tome de la série de Stephane Heuet à travers à peine 70 planches dessinées qui répondent au principe d'un récit chronologique, d'une histoire qui avance de manière linéaire jusqu'à la dernière case. Comme nous l'avons déjà souligné, un tel cheminement ne ressemble en rien à l'écriture proustienne. On est donc en droit de se demander comment, malgré ce principe fondamental d'économie, le roman graphique réussit-il tout de même son pari de rester fidèle au texte littéraire?

D'une part, comme l'a relevé Marie-Hélène Gobin (2006: 70-73) dans son «Proust en BD, que dirait Baudelaire ", où elle met en face à face les structures narratives des deux récits (littéraire chez Proust et du roman graphique), on s'aperçoit tout de suite d'une omission majeure dans le nouvel agencement du récit prévu par Stéphane Heuet. L'univers séquentiel ne respecte pas l'unité du cycle proustien ${ }^{2}$ : après la parution de Combray en BD, on remarque l'absence d'Un amour de Swann (qui ne paraît qu'en 2008) et de Nom de pays (qui ne paraitra en BD sous la plume de Heuet qu'en 2013). Le Combray de Heuet sera d'abord suivi des 2 tomes d'A l'ombre des jeunes filles en Fleurs (qui paraissent tout de suite après Combray, en 2002). Cette dislocation de la trame originale du récit proustien ne sera corrigée qu'en 2013 avec la publication d'un tome intégral intitulé $\mathrm{Du}$ côté de chez Swann, aujourd'hui disponible parallèlement aux trois volumes isolés du cycle de Swann, d'abord disloqué.

Cette omission-dislocation s'explique cependant, dans l'univers linéaire de la planche, si l'on considère le principe d'économie de lecture qu'elle engage. Un amour de Swann place en effet, dans le texte proustien, le lecteur devant une difficulté majeure. Ce volume représente une sorte d'anachronie dans la linéarité du roman puisqu'il fait intervenir une troisième instance narrative qui alterne subitement avec les figures du jeune Marcel enfant et du narrateur adulte campées dans le premier tome de Combray: Swann, cet autre je, à la fois double et intermédiaire fait irruption au risque de gêner le principe d'identification établi jusqu'ici entre Proust et son lecteur. L'économie consiste ici en un principe de dislocations et de coupures de la structure narrative initiale, qui a pour but d'assurer une lecture efficace de l'œuvre littéraire dans le roman graphique. Paradoxalement, les blancs deviennent des aides pour le lecteur de Proust, en particulier pour le lecteur néophyte, qui pénètre ainsi plus facilement le plan de lecture global de l'œuvre ${ }^{3}$.

13 Toutefois, ce raccourci est dans le même temps compensé par tout un effet de rappels, un phénomène de redondance, observable au niveau de certaines icônes. Ces dernières réalisent, de manière métaphorique, la soudure entre la trame narrative initiale du roman proustien et la trame narrative très fortement elliptique de l'art séquentiel. 
14 Par exemple, la BD de Combray s'ouvre et se ferme sur une icône qui représente la fenêtre du coucher qui est initialement associée chez Proust aux peurs du coucher du jeune Marcel. Mais, la sémantique de l'image change très fortement d'une scène à l'autre, et dépasse le texte. Sur la première planche de la BD de Heuet, la fenêtre est fermée (conformément à l'incipit du roman). Or, on retrouve cette icône sur la dernière planche de la BD de Heuet. Mais le volume se clôt, si l'on peut dire, non pas sur une fenêtre fermée, mais sur l'image d'une fenêtre désormais ouverte. Or, dans La Recherche (1987 : 184), on ne fait pas allusion à une fenêtre ouverte. La fenêtre est bel et bien fermée. La scène se passe certes au lever du jour, mais le jeu de lumière que décrit Proust se fait dans ce passage à travers l'obscurité diffuse d'un rideau fermé, une opacité qui sert alors de surface de projection à l'imagination féconde du narrateur :

Mais à peine le jour - et non plus le reflet d'une dernière braise sur une tringle de cuivre que j'avais pris pour lui - traçait-il dans l'obscurité, et comme à la craie, sa première raie blanche et rectificative, que la fenêtre avec ses rideaux quittait le cadre de la porte où je l'avais située par erreur, tandis que, pour lui faire place, le bureau que ma mémoire avait maladroitement installé là se sauvait à toute vitesse, poussant devant lui la cheminée et écartant le mur mitoyen du couloir; une courette régnait à l'endroit où, il y a un instant encore, s'étendait le cabinet de toilette, et la demeure que j'avais rebâtie dans les ténèbres était allée rejoindre les demeures entrevues dans le tourbillon du réveil, mise en fuite par ce pâle signe qu'avait tracé au-dessus des rideaux le doigt levé du jour.

Le décalage sémantique que Heuet introduit au niveau de la répétition de l'icône est un appel en direction de son lecteur. Ce procédé force à anticiper, à établir un lien, au seuil du premier volume de Combray, préfigurant l'évolution à venir dans les différents tomes de La Recherche. La dernière image annonce, en effet, le déploiement d'un moi et de la mémoire sensible (signalisé ici à travers la position désormais ouverte de la fenêtre), qui ne va pourtant pouvoir se déployer chez Proust que dans le tout dernier volume de La Recherche, intitulé Le Temps retrouvé .

Ce raccourci narratif établit par l'image a en outre une fonction essentielle : cette dernière assure la mise en place d'une très forte tension narrative telle que Baroni (2007) la définit. A travers la répétition de l'image, le lecteur de BD est amené à faire des diagnostics ainsi que des pronostics sur le déroulement futur - et la fin - de l'histoire à venir. L'image l'engage dans sa lecture, et le guide sur la voie de son interprétation. Toutefois, c'est au lecteur d'en fixer la valeur.

En effet l'icône graphique de la fenêtre (cf image 1 : fermée sur la première page, puis, cf. image 2 : ouverte sur la dernière page) dépeint un instantané, un moment précis de l'histoire qui contraste très fortement avec le « longtemps » du récitatif proustien situé en marge dans la case couleur coquille d'œuf (cf. image 1). A travers cet encadrement, un art de la métalepse se met en place, faisant communiquer texte et image, et qui va permettre au lecteur de la BD de Heuet d'apprécier lui-même l'expérience de la profondeur du temps et de l'espace proustiens. Le contraste, en effet, est évident entre le volet fermé au début de Combray et le volet ouvert à la fin de la BD de Heuet. Comme nous l'avons dit, cela est une opposition voulue, scénarisée par Heuet, alors qu'elle n'est pas présente dans le texte de Proust. Ce raccourci narratif par l'image expose la confrontation de concepts qui sont fondamentaux pour la compréhension de l'œuvre proustienne: l'intérieur et l'extérieur, le visible et l'invisible se retrouvent ici très nettement associés à l'intériorité d'un «je » caché derrière l'opacité du volet (dans le cas de la première scène). À l'incipit du roman littéraire comme du roman graphique, la 
notion d'intérieur/d'intériorité renvoie à une notion d'oppression et d'enfermement. Mais, à la fin du premier tome de la BD de Heuet, s'annonce déjà la promesse d'un dépassement: souffle, air, liberté; les mêmes concepts se retrouvent retournés en condition de l'art et de la représentation, en promesse de l'éveil d'une conscience qui ne se fera chez Proust, comme nous l'avons dit, que bien plus tard dans La Recherche.

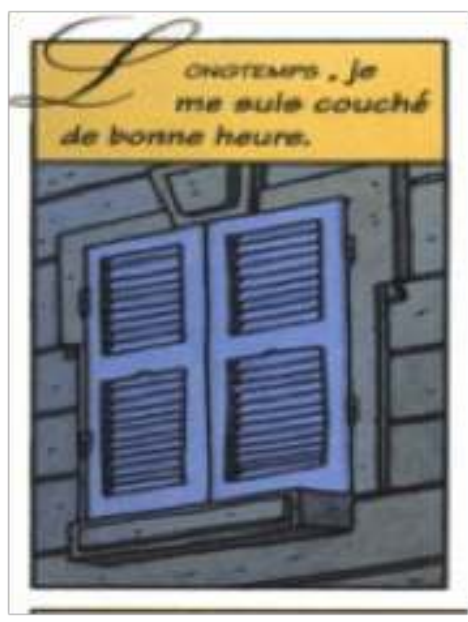

A LA RECHERCHE dU TEMPS PERDU DE MARCEL PROUST, TOME 1, ADAPTÉ PAR STÉPHANE HEUt (C) EDITIONS DELCOURT - 1998

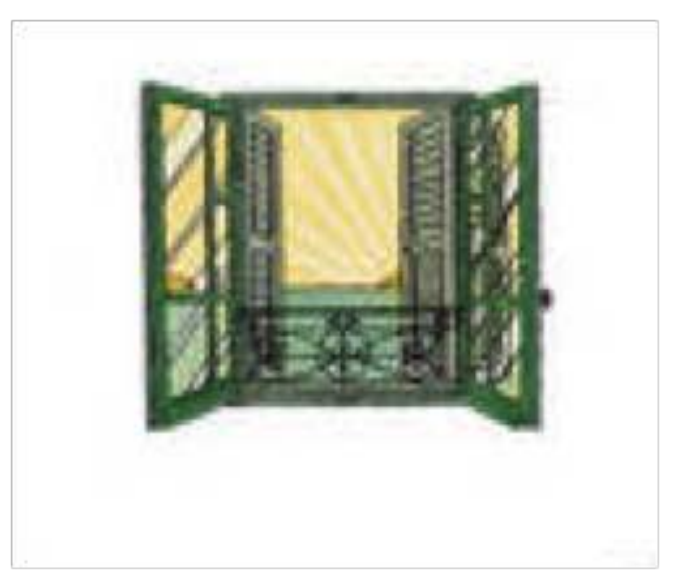

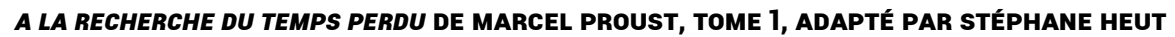
(C) EDITIONS DELCOURT - 1998

Les zones de coupes et de brisures qui affectent l'agencement de chacune des cases forcent donc le lecteur de BD à établir lui-même des correspondances avec des parties encore absentes de l'œuvre. Il participe ainsi à la reconstitution active d'un nouveau plan de lecture, qui - et cela est toute la réussite de Heuet - lui permet de revivre et de s'approprier, par la lecture, les mécanismes de la réminiscence proustienne. Le principe d'économie de lecture inhérent au genre de la BD coïncide alors ici avec un des principaux impératifs de l'art littéraire : il s'agit d'assurer une véritable esthétique de la réception permettant de recréer l'unité de la séquence. En effet, comme nous avons pu l'observer, il n'y a pas ici à proprement parler un travail de dislocation de la trame narrative littéraire, mais plutôt un très fort principe d'hybridation de ses constituants ; si bien que, si dislocation il y a dans l'univers séquentiel de la BD, cette dislocation est une dislocation étonnamment féconde qui pose son lecteur devant un important travail herméneutique. 


\section{Économie de lecture et recréation/récréation proustienne}

19 Stéphane Heuet (2012) le rappelle dans l'auto-analyse qu'il fait de son œuvre graphique : lors de la transformation du texte littéraire en BD, ce dernier finalement se ramifie, le texte initial peut en effet « avoir quatre destinées » :

20 - il peut devenir dialogues (dans les bulles)

21 - être transformé en voix off (il s'agit dans la BD de Heuet du texte marqué dans les cases de couleur coquille d'oeuf que l'auteur a lui-même pensé en référence à Black et Mortimer. Ce texte fonctionne, selon Heuet, comme "un récitatif», «c'est l'imparfait itératif au texte »)

22 - il peut devenir image (sous forme de dessins dans les cases)

23 - ou bien encore disparaître : sort «terrible » comme le souligne Heuet; mais, par principe d'économie et souci d'efficacité, la BD se doit de privilégier des phrases « utiles » plutôt que de rendre les volutes de la poésie proustienne.

24 Heuet met donc automatiquement en place un nouveau plan de lecture de l'œuvre proustienne. Il propose une nouvelle disposition du texte qui répond, selon ses dires, à deux attentes principales : d'une part, assurer le plaisir de lecture, et, d'autre part, rester fidèle au texte. Dans l'auto-analyse qu'il fait de son travail, Heuet (2012: 149) avance l'exemple des bulles qu'il organise avec beaucoup de minutie. Comme il le souligne, chaque fois, c'est une esthétique de la réception qui motive son choix concernant leur emplacement par rapport au reste du texte et des images:

Je travaille leur emplacement, (...). Leur situation les unes par rapport aux autres est aussi pour moi très importante. Je ne veux pas, comme j'en ai parfois le déplaisir en tant que lecteur, lire une réponse avant une question, ou simplement voir le rythme de la lecture, sa fluidité, mis à mal par une hésitation sur la bulle à lire en premier.

Heuet précise que ce nouveau travail de soudure « doit être invisible ». Or, sur ce point, l'auteur de bande dessinée reprend une technique caractéristique de l'écriture proustienne ${ }^{5}$. Celle-ci est particulièrement visible à la lecture des manuscrits de $L a$ Recherche. Entre le prétexte et la version définitive, Proust se livre, en effet, à tout un travail d'effacement, de gommages délibérés au niveau des liens logiques qui unissaient à l'origine l'effet à la cause d'un événement. De cette manière, le texte proustien renforce toujours, dans sa version définitive, une impression de sauts d'humeur livrés à la pensée et à travers lesquels sont lecteurs doit se frayer un chemin, tenter d'avancer sa propre explication logique. L'écriture proustienne mime au plus près les méandres dessinés lors de la remontée du souvenir à travers le jeu de la mémoire sensible. Le lecteur de Proust est plus que jamais engagé dans sa lecture dans un acte de reconstruction et de déchiffrement en tous points semblable au cheminement du lecteur de Heuet, qui doit pallier les blancs afin de «restituer le temps et le mouvement» dans l'univers séquentiel de la bande (MCCloud 2007: 77), et, dans le même temps, par ce biais, recrée à son tour, par le jeu de son imagination, la linéarité et le souffle initial de la phrase proustienne.

Pour bien comprendre ce parallèle, arrêtons-nous quelques instants sur l'analyse du passage bien connu de la madeleine (Proust 1987: 44-47), dans lequel le lecteur 
proustien a généralement l'habitude de se laisser glisser, avant d'observer sa transposition graphique dans la BD de Stéphane Heuet:

Il y avait déjà bien des années que, de Combray, tout ce qui n'était pas le théâtre et le drame de mon coucher n'existait plus pour moi, quand un jour d'hiver, comme je rentrais à la maison, ma mère, voyant que j'avais froid, me proposa de me faire prendre, contre mon habitude, un peu de thé. Je refusai d'abord et, je ne sais pourquoi, me ravisai. Elle envoya chercher un de ces gâteaux courts et dodus appelés Petites Madeleines qui semblaient avoir été moulées dans la valve rainurée d'une coquille de Saint-Jacques. Et bientôt, machinalement, accablé par la morne journée et la perspective d'un triste lendemain, je portai à mes lèvres une cuillerée du thé où j'avais laissé s'amollir un morceau de madeleine. Mais à l'instant même où la gorgée mêlée des miettes du gâteau toucha mon palais, je tressaillis, attentif à ce qui se passait d'extraordinaire en moi. Un plaisir délicieux m'avait envahi, isolé, sans la notion de sa cause. Il m'avait aussitôt rendu les vicissitudes de la vie indifférentes, ses désastres inoffensifs, sa brièveté illusoire, de la même façon qu'opère l'amour, en me remplissant d'une essence précieuse: ou plutôt cette essence n'était pas en moi, elle était moi. J'avais cessé de me sentir médiocre, contingent, mortel. D'où avait pu me venir cette puissante joie? Je sentais qu'elle était liée au goût du thé et du gâteau, mais qu'elle le dépassait infiniment, ne devait pas être de même nature. D'où venait-elle? Que signifiait-elle? Où l'appréhender? Je bois une seconde gorgée où je ne trouve rien de plus que dans la première, une troisième qui m'apporte un peu moins que la seconde. Il est temps que je m'arrête, la vertu du breuvage semble diminuer. Il est clair que la vérité que je cherche n'est pas en lui, mais en moi. Il l'y a éveillée, mais ne la connait pas, et ne peut que répéter indéfiniment, avec de moins en moins de force, ce même témoignage que je ne sais pas interpréter et que je veux au moins pouvoir lui redemander et retrouver intact, à ma disposition, tout à l'heure, pour un éclaircissement décisif. Je pose la tasse et me tourne vers mon esprit. C'est à lui de trouver la vérité. Mais comment? Grave incertitude, toutes les fois que l'esprit se sent dépassé par lui-même ; quand lui, le chercheur, est tout ensemble le pays obscur où il doit chercher et où tout son bagage ne lui sera de rien. Chercher? pas seulement: créer. Il est en face de quelque chose qui n'est pas encore et que seul il peut réaliser, puis faire entrer dans sa lumière (...)

Et dès que j'eus reconnu le goût du morceau de madeleine trempé dans le tilleul que me donnait ma tante (quoique je ne susse pas encore et dusse remettre à bien plus tard de découvrir pourquoi ce souvenir me rendait si heureux), aussitôt la vieille maison grise sur la rue, où était sa chambre, vint comme un décor de théâtre s'appliquer au petit pavillon donnant sur le jardin, qu'on avait construit pour mes parents sur ses derrières (ce pan tronqué que seul j'avais revu jusque-là); et avec la maison, la ville, la Place où on m'envoyait avant déjeuner, les rues où j'allais faire des courses depuis le matin jusqu'au soir et par tous les temps, les chemins qu'on prenait si le temps était beau. Et comme dans ce jeu où les Japonais s'amusent à tremper dans un bol de porcelaine rempli d'eau de petits morceaux de papier jusque-là indistincts qui, à peine y sont-ils plongés s'étirent, se contournent, se colorent, se différencient, deviennent des fleurs, des maisons, des personnages consistants et reconnaissables, de même maintenant toutes les fleurs de notre jardin et celles du parc de M. Swann, et les nymphéas de la Vivonne, et les bonnes gens du village et leurs petits logis et l'église et tout Combray et ses environs, tout cela qui prend forme et solidité, est sorti, ville et jardins, de ma tasse de thé. l'ensemble de La Recherche proustienne: sur trois planches successives, son lecteur redécouvre en effet un plaisir et une esthétique de lecture qui reflète parfaitement le jeu esthétique mis en place par Proust dans l'épisode de la madeleine. 
Voir les annexes 1 (image 3), 2 (image 4) et 3 (image 5), issues de $A$ la recherche $d u$ temps perdu de Marcel Proust, tome 1, adapté par Stéphane Heuet. (C) Editions Delcourt - 1998

Dans cette séquence s'étalant sur trois planches successives, notre regard est amené à glisser d'une image à l'autre, porté par le mouvement conducteur - en forme de volute - que décrit la fumée qui s'échappe de la tasse de thé (cf. image 3). Celle-ci se trouve à l'intérieur d'une série de quatre cases (situées en haut à droite de la planche) qui constituent la matrice narrative initiale, le noyau séquentiel de cet épisode, préludant - chez Heuet comme chez Proust - au long développement auquel va donner lieu l'expérience gustative et olfactive de la madeleine. Le graphisme choisi par Heuet rend très bien l'effet de "tourbillon" ascendant évoqué dans le texte proustien. La fumée blanche, qui s'échappe de la micro-séquence initiale de la madeleine plongée dans la tasse de thé, traverse les blancs des pages, relie les cases entre elles et fait fusionner les trois planches en une unité complète. Surtout, à travers son graphisme et l'ordonnance des cases, Heuet prend soin de rendre - et de conduire son lecteur à travers - le fonctionnement caractéristique de la mémoire sensible: il s'agit d'un mécanisme de remontée à la fois centrifuge et centripète du souvenir, déjà bien connu des premiers neurologistes contemporains de Proust (Bogoussalavsky/Walunsinski 2008 : 246) et ici brillamment mis en scène par le graphisme de Heuet. Le lecteur qui s'engage dans le déchiffrement de ces planches est en effet amené à reconstruire ce double mouvement. Son regard est conduit par les volutes de la fumée qui s'échappe de la macro-proposition initale de la tasse de la tasse de thé (cf. image 3). La fumée se déploie et sillonne ensuite à travers les blancs des pages. Mais, parallèlement, la case située au centre de la première page - qui dépeint le narrateur les yeux ouverts, en sujet conscient - constitue une seconde macro-proposition initiale autour de laquelle tout l'épisode vient se solidariser. Elle représente le point de départ du travail d'introspection sensorielle mis en branle dans l'expérience de la madeleine. Chez Proust, ce moment est décrit sur la longueur de tout un paragraphe saturé d'un flot de questions. Dans la BD de Heuet, nous retrouvons ce passage résumé à l'intérieur d'une case de grandeur minimale mais qui, en raison de sa construction très fortement métonymique, est saturée de sens. La synecdoque s'y déploie en effet sur trois niveaux. L'espace-temps du récit se creuse : l'acuité présente du narrateur éveillé est renvoyée à l'intensité du questionnement portant sur l'origine indéterminée de la sensation symbolisée par le point d'interrogation. À un troisième degré, le court récit itératif tiré du roman proustien, situé en bas de la case (« Un plaisir délicieux m’avait envahi, isolé, sans la notion de sa cause ») encadre l'ensemble, ramenant explicitement le déploiement à venir de ce questionnement à la présence d'une conscience "isolée ». Pour le lecteur de Heuet, la lecture de ces trois planches exige donc un acte de lecture complexe mettant en relation ces trois niveaux. L'agencement de l'univers séquentiel l'invite à revivre, pas à pas, à travers sa lecture le cheminement de la mémoire involontaire initié dans l'épisode de la madeleine. À son tour, il reconstruit, comble les espaces, anticipe les blancs et revient sur ses pas : il suit les traces laissées par « la ligne claire » voulue par Heuet. 


\section{La ligne claire}

des pages et qui dirige la lecture à travers l'espace séquentiel de la BD. Scott MCcloud les qualifie de «blancs magiques » (2007: 100), car, selon lui, la performance de l'icône réside très précisément dans ces vides, ces espaces, ces effets de coupures laissés entre les cases $^{6}$. De même, le blanc narratif créé par l'omission textuelle, phénomène nécessaire dans la transposition du texte littéraire dans l'univers séquentiel, est en quelque sorte comblé, rempli par le blanc de l'icône, par les espaces qui - de manière paradoxale - relient les cases entre elles, et signalisent au lecteur que c'est là où se joue son travail de reconstitution active, de re-création du texte littéraire qu'il a à prendre en charge et qui est le fondement même du plaisir esthétique. Cet espace de re-création est, dans la BD, un espace fort ludique, car le neuvième art est un espace dans lequel auteur et lecteur prennent certaines libertés, adoptent une posture déjà distanciée par rapport au texte original. Les blancs qui organisent la disposition de la page l'affirment et signalisent immédiatement au lecteur son engagement nécessaire dans l'acte de reconstitution de l'histoire.

Pour favoriser ce fait, Heuet a, comme nous l'avons vu, opté pour une chronologie simple. Il a surtout fait le choix d'une typographie à la Tintin qui a au départ dérouté les experts et a pu leur faire croire qu'il s'agissait bien ici d'un "Proust au rabais ». Heuet était lui-même conscient du danger et des critiques auxquelles il allait s'exposer. Il affirme: " pour dessiner les personnages, j'ai fait le choix improbable de la ligne claire» (2012 : 150). Mais son choix était motivé par le souhait de laisser le graphisme des personnages le plus abstrait possible afin de favoriser, de renforcer le principe d'identification entre son lecteur et ses personnages. L'art de la BD fait très fortement appel à un plaisir esthétique basé avant tout sur l'affect et le plaisir de la réception chez son lecteur.

L'espace de la BD est un genre tout particulièrement ouvert aux phénomènes de coopération interprétative tels que les a décrits Umberto Eco (1989) à la suite des travaux de Wolfgang Iser et Hans-Robert Jauss. Dans l'art séquentiel, l'acte de lecture est, plus que jamais, un acte de re-création qui exige la participation active de son lecteur (cf. MCcloud 2007 : 77) :

L'ellipse volontaire que pratique le lecteur est le moyen fondamental par lequel la bande dessinée peut restituer le temps et le mouvement.

L'ellipse dans la bande dessinée favorise entre le lecteur et le créateur une intimité qui n'est surpassée que par le monde de l'écrit, elle est à l'origine d'un contrat silencieux et secret.

Le travail de déchiffrage, qui consiste en premier lieu à anticiper les blancs, les coupures visibles au niveau des espaces perceptibles sur la planche est, comme nous venons de le voir, en partie guidé, préfiguré par le travail d'agencement des vignettes prévu par l'auteur. Mais, en contre-partie, la BD laisse une très grande place et fait directement appel au monde propre à son lecteur. Heuet le souligne : la BD convoque l'« inconscient » du lecteur, qui doit « se sentir en connivence » et devenir, au fil de sa lecture, le lecteur de soi-même : «il doit sentir un monde culturel connu, ces indices seront propres à chacun, certains reconnaitront d'autres pas, chacun se créant son monde à partir de celui de Proust » $(2012: 150)$.

Cahiers de Narratologie, 31 | 2016 

raison de cette plasticité de l'image proustienne, l'adaptation de cette œuvre phare de la littérature française en BD était une entreprise risquée mais prometteuse, un pari que Stéphane Heuet a aujourd'hui très largement remporté. On retrouve chez Heuet comme chez Proust les mêmes visages-écrans des personnages, les mêmes surfacesplanes et iconiques qui donnent à la couleur toute sa valeur métaphorique (un petit pan de mur jaune, le visage d'Odette...). Mais avant tout, c'est l'esthétique de la réception que le genre de la BD met en place, qui permet au lecteur de Heuet de renouer avec l'expérience de la mémoire involontaire qui fonde l'essence de La Recherche. Chez Heuet comme chez Proust, des sortes d'images-hiéroglyphes font directement appel au lecteur, et exigent de lui - en particulier dans l'économie de la séquence graphique - un travail d'autant plus intense des sens et de la réminiscence. L'esthétique de la réception, mise en place dans l'univers restreint de la $\mathrm{BD}$, force le lecteur à participer activement, à s'engager pleinement dans la reconstruction de l'expérience proustienne, sauf que, si chez Proust, la réminiscence passe d'abord par le goût et l'odorat, chez Heuet, elle passe en premier lieu par la vue.

Cahiers de Narratologie, 31 | 2016 


\section{BIBLIOGRAPHY}

Baetens, J. (2009), «Littérature et bande dessinée. Enjeux et limites ». Cahiers de Narratologie [En ligne], 16 | 2009, mis en ligne le 25 mai 2009, consulté le 25 juin 2016.

Baroni, R. (2007), La tension narrative. Paris : Seuil.

Bizub, E. (2005), Proust et le moi divisé. Paris : Droz.

Bogousslavsky/Walusinski (2008), « À la recherche du neuropsychiatre perdu : Paul Sollier (1861-1833)». Revue neurologique, vol. 164, n HS3 (sept. 2008).

Fresnault-Deruelle, P. (1972), Dessins et Bulles. Paris : Bordas.

Fresnault-Deruelle, P. (1977), Récits et discours par la bande. Paris : Hachette.

Gobin, M.-H. (2006),_Proust en BD, que dirait Baudelaire. Paris : Editions Connaissances et Savoirs.

Heuet, S. (1998), Combray. A la recherche du temps perdu. Paris : Declourt.

Heuet, S. (2012), « La Recherche en BD ». Revue Marcel Proust Aujourd'hui n9.

Iser, W. (1995), L'acte de lecture. Théorie de l'effet esthétique. Paris : Mardaga.

Jauss, H.-R. (1978), Pour une esthétique de la réception. Paris : Gallimard.

MCCloud, S. (2007), L'art invisible. Paris : Delcourt.

Proust, M. (1987), A la recherche du temps perdu I. Paris : Gallimard.

Umberto, E. (1972), « Epilogue ». L’Art de la bande dessinée, ed. par Walter Herdeg/David Pascal. Zürich : The Graphis Press.

Umberto, E. (1989), Lector in fabula. Le rôle du lecteur, ou, la cooperation interprétative dans les textes narratifs. Paris : Gallimard.

\section{NOTES}

1. Voir l'article de libération: «Longtemps, j'ai bullé de bonne heure », en réponse à la critique assassine parue lors de la sortie de Combray dans le Figaro, consultable en ligne (http:// next.liberation.fr/livres/1998/09/03/longtemps-j-ai-bulle-de-bonne-heure-en-mettant-larecherche-en-cases-et-en-bulles-stephane-heuet-s-a_247398): «En mettant "La Recherche" en cases et en bulles, Stéphane Heuet s'attire les foudres de l'orthodoxie de Proustiens ».

2. Le premier tome d'A la Recherche du temps perdu se compose de trois parties: Combray, Un amour de Swann et Nom de pays.

3. Citons ici en exemple l'incipit bien connu de Combray: les «évocations tournoyantes et confuses » lâchées par la mémoire d'un corps au moment du coucher sont décrites sur sept pages dans l'édition Pléiade de La Recherche, alors qu'elles se trouvent résumées sur une seule planche (p.3) à l'ouverture de la BD de Combray.

4. La Recherche est un roman portant sur la crise d'une vocation d'écrivain. Celle-ci ne sera surmontée que dans le dernier volume de La Recherche, après une cure; la révélation littéraire qui se fera après la cure ira alors de pair avec une autre révélation : celle des mécanismes du moi inconscient et du fonctionnement de la mémoire sensible. Voir à ce sujet : Bizub (2005).

5. Dans un article intitulé «A propos du style de Flaubert » paru dans La Nouvelle Revue Française en 1920, Marcel Proust loue la technique du blanc et l'absence de transition caractéristique du 
style de Flaubert et qui lui servira de modèle, car, en l'absence de transition, dit-il, « soudain la mesure du temps devenant au lieu de quarts d'heure, des années, des décades ».

6. "C'est pourquoi je pense que c'est une erreur de ne voir en la bande dessinée qu'un genre hybride entre les arts graphiques et le récit littéraire. Ce qui se passe entre les cases est une sorte de magie que seule la bande dessinée sait créer ».

\section{ABSTRACTS}

The transposition of Proust's Remembrance of Things Past into a graphic novel is an operation based on a fundamental principle of narrative economy. The comic-strip request to make choices, to transcribe the proustian text in a space made of serial cuts and broken lines : Heuet's new scenario implies a central act of reduction, a deconstruction-reconstruction of literary narrative. This article will highlight that instead of betraying the proustian text, the new narrative imposed by space of the storyboard reminds faithfully of the proustian aesthetic and leads the comic reader on the way to his own madeleine.

La transposition de La Recherche en BD repose sur un principe d'économie fondamental. Le principe de réduction qui s'engage dans l'espace réduit de la planche demande de faire des choix, de ne pas pouvoir tout dire, mais bien de transcrire par l'image la phrase proustienne. La nouvelle scénarisation de La Recherche entreprise par Stéphane Heuet implique donc un acte de déconstruction-reconstruction de la narrativité littéraire au sein d'un espace sériel fait de coupures, de brisures et de traits.

Cet article analyse l'apport de cette nouvelle scénarisation sous l'aspect de son économie narrative. En soulignant le parallèle qui existe entre le principe de stratification sérielle dans l'espace restreint de la BD et les mécanismes de remontée du souvenir mis en lumière par Proust, nous verrons combien le principe de réduction imposé par l'art séquentiel exige la formation d'un pacte de lecture très fortement récréatif et fécond, fort proche de l'esthétique proustienne, qui permet ainsi au lecteur de BD d'accéder à sa propre madeleine.

\section{INDEX}

Mots-clés: Proust Marcel, Heuet Stéphane, À la recherche du temps perdu, bande dessinée, esthétique de la réception, lecture, économie

Chronological index: XXe siècle

Geographical index: France

\section{AUTHOR}

JULIE MÜLLER

Université de Hambourg 